\title{
Modeling Software Platform of Multi-Organization Core Based on Voxels Interpolation Method
}

\author{
Niu-Wenjie ${ }^{1}{ }^{*}$, Liu-He $^{1}$, Wang-Xiaobin ${ }^{1}$, Xu-Qingqing ${ }^{1}, \mathrm{Xu}$ Ying $^{2}$, Zheng-Shipo $^{1}$, \\ Li-Hui ${ }^{1}$ and Bai-Yongtao ${ }^{1}$ \\ ${ }^{1}$ Institute of Mechanical and Electronic Engineering, China University of \\ Petroleum (East China), Qingdao 266580, China \\ ${ }^{2}$ Sanying Precision Instruments Ltd, Dongying 257091, China \\ niuwj67@126.com
}

\begin{abstract}
The multi-organization modeling technology is widely used in medical field. But there is no application in reservoir cores which correlative study is also rare. In this paper, a new segmentation modeling method of multi-organization core based on labeled voxels interpolation is proposed. Firstly, the surface model of core is constructed by using the image processing technology and surface model reconstructed technology based on the CT images of the core. Then, the tetrahedral model of core is constructed by using constrained Delaunay triangulation algorithm. The information of model elements is obtained through labeling data information and interpolating model information on the tetrahedron model. Finally, the model units of different organization were segmented to organization model with a single attribute of the reservoir core which realized the multiorganization core reconstruction. The visualization and interaction functions of CT images and core models are realized based on the OpenGL technology. The software is good for attempt of multi-organization core modeling which provides data support for the following simulation experiments and finite element analyses.
\end{abstract}

Keywords: Digital core; Multi-Organization; Model reconstruction; Tetrahedral segmentation

\section{Introduction}

In this paper, the multi-organization of the core means different components or different phases (such as liquid, gas, etc.) of the core. The core multi-organization has an extremely rough facial structure, very detailed surface and complex interfaces between organizations, which is quite different from the medical multi-organization. Nowadays, the multi-organization modeling technology is widely used in medical fields [1-2]. In foreign countries, the correlation study of the technology of body sectional images and multi-organization body reconstruction has been proposed throughout a few decades, in which the SimBio project and GEMSS project [1] are the most representative. Some representative commercial software such as Amira and SimPleware, both have achieved the image reconstruction and realized some functions such as image preprocessing, surface mesh generation, volume mesh generation and material properties replication [1].

In China, the study on multi-organization modeling is still in its initial stage. A representative method to build tetrahedral mesh directly by classifying medical image data was proposed by Yang Xiaosong of [3] Tsinghua University. This method can generate multi-organization tetrahedral model with adaptive density.

In the field of petroleum geology, CT technology is mainly used for the rapid and nondestructive testing of core to calculate some physical parameters including core density, porosity and saturation. CT technology is also used to reconstruct the core model to do some numerical analyses based on CT images. However, there is no relative 
application of multi-organization modeling technology of core based on CT images or relevant multi-organization core modeling software. So we developed a visual modeling software which is aiming at multi-organization core based on core scanning images by using CT technology. This software achieves the visual modeling of multi-organization core which can offer easy operations reconstruct and observe the multi-organization core model and also provides an amity interactive interface to users to operate the visual model.

\section{Modeling Platform Designed for Multi-organization Core}

\subsection{Platform framework}

The platform of multi-organization core modeling software is developed based on C++ language, with the help of OpenGL graphics library to display model and achieve the interactive operation. The main functions of the platform are as follows:

1. CT image processing: image reading, image writing, image noise reduction, image filtering, image segmentation and voxels extraction;

2. Model reconstruction: iso-surface extraction, model simplification, model smoothing and constrained tetrahedron subdivision;

3. Multi-organization segmentation: labeling multi-organization voxels, getting voxels interpolation, boundary segmentation and organization extraction;

4. Model display and interactive operation.

\subsection{Data Structure}

In this paper, the data structure is based on linked list, including "Link" linked list and "LinkNode" linked list. In the process of modeling, the treated data types include CT images, core voxels, core surface model, core tetrahedron model, core multi-organization model. The treated data types are shown in Figure 1.

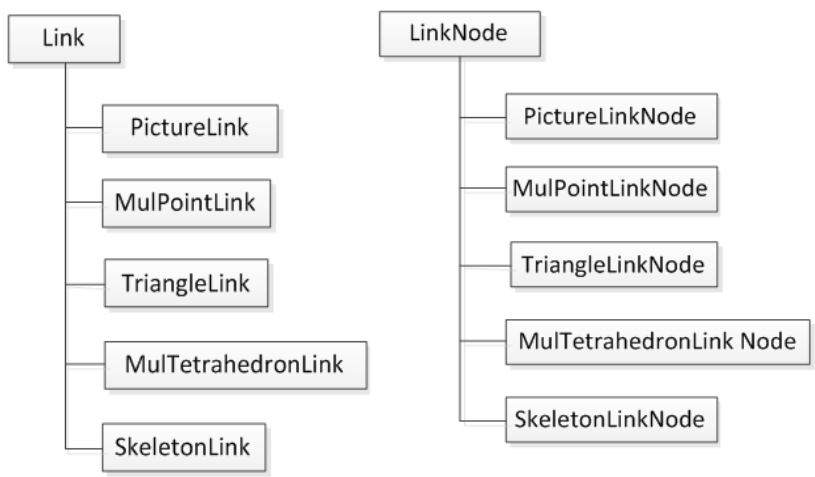

Figure 1. Class Inheritance

In order to guarantee the accurate topology of the model, the topological relations between tetrahedral elements are established, as shown in Figure 2. 


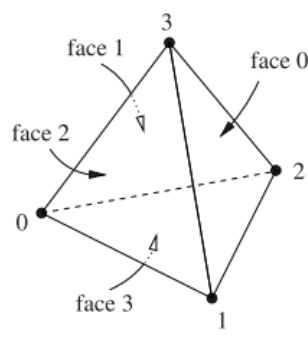

(a)

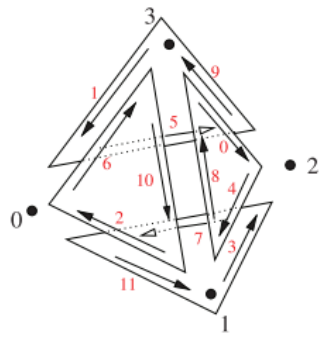

(b)

Figure 2. Topology of Tetrahedron

As is shown in Figure 2a, we use numbers 0, 1, 2, 3 to denote the four vertices of the tetrahedron, use face 0 , face1, face2, face 3 to denote the four triangular faces of the tetrahedron and denote the index of the triangular face equal to the index of its opposite vertex. The definition of the adjacency relation between tetrahedrons is shown in Figure 3b: the normal vector direction of each surface follows the left-hand screw rule pointing external and records the tetrahedron number.

\subsection{Modeling Method of Multi-Organization Core}

\subsubsection{Algorithm Flow}

In this paper, we reconstruct the multi-organization core model based on tetrahedral modeling and voxels. First, the CT images (including image noise reduction and image segmentation [7-8], etc.) should be preprocessed to obtain the voxels of multiorganization core; then, reconstruct surface model using MC algorithm [9-11]; finally, reconstruct tetrahedron model using constrained tetrahedral subdivision method [12-14].

The multi-organization model is reconstructed after boundary division between different organizations based on the accurate tetrahedral mesh. According to the multi-organization model information, the size, position, digital parameters and relative position relationship of organizations are determined. The concrete operation flow is shown in Figure 3:

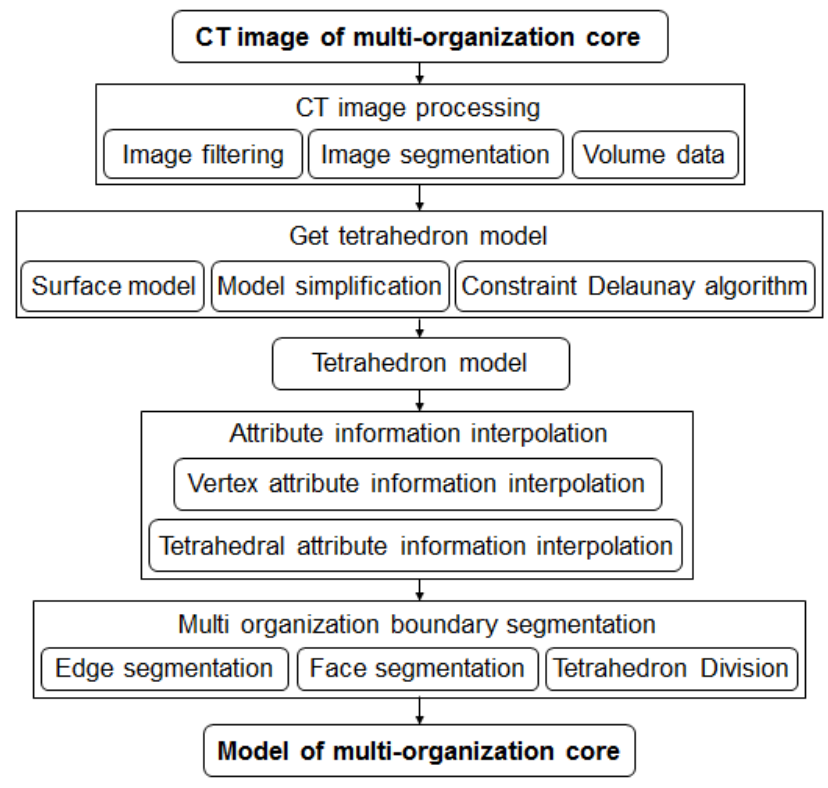

Figure 3. Flow Chart of Modeling Method of Multi Organization Core by Labeling Voxels 


\subsubsection{Vertex Attribute Interpolation}

The tetrahedral vertices of core model include voxels information. There is a one - to one match between voxel and tetrahedral vertex without the interpolation points on the model surface. Therefore, the interpolation of tetrahedral vertices attributes is realized by finding the corresponding voxels and copying the organization attribute information into the tetrahedral vertices.

\subsubsection{Tetrahedral Attribute Interpolation}

After the vertices attributes are determined, a series of tetrahedral elements with vertices attributes are obtained. We propose an algorithm to divide the tetrahedral elements into three types according to the information of vertices attributes:

The first type: the four vertices of the tetrahedron are without attribute information, as is shown in Figure 5a. This kind of tetrahedrons appears in the corner of the model and these tetrahedrons are considered to have the same attribute as their adjacent tetrahedrons. For this, we insert the information of vertices attributes of their adjacent tetrahedrons into the first type tetrahedrons.

The second type: some vertices of the tetrahedron are with attributes but others without, as is shown in Figure 5 (c, d, b). This kind of tetrahedral vertices contains the surface vertices and voxels points. For this, we insert the vertex attribute information into the vertices without attribute.

The third type: the four vertices of the tetrahedron all have attribute values, as is shown in Figure 4 (f, g, e, h, j). The vertices attributes of this kind of tetrahedron are divided into two types: single attribute tetrahedron (which is shown in Figure 4(e)) and multiorganization tetrahedron (which is shown in Figure 4 (f, h, g, j)). The tetrahedrons of different vertices attributes need to be carried out for the next step of multi-organization boundary segmentation.

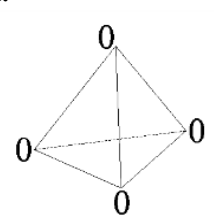

(a)

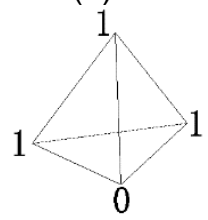

(d)

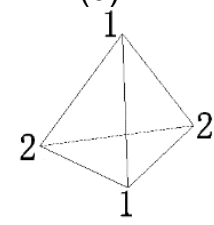

(g)

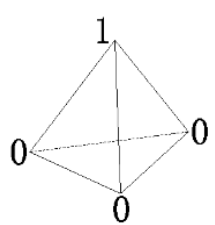

(b)

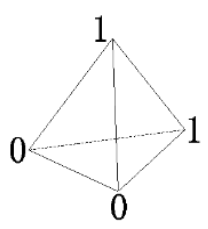

(c)<smiles>C1C2C3C1C23</smiles>

(e)<smiles>C1C2C3C1C23</smiles>

(h)

(j)

Figure 4. Property of Vertex of Tetrahedron

\subsubsection{Boundary Tetrahedron Segmentation}

Boundary tetrahedron segmentation means dividing the tetrahedral elements whose attributes are not single after interpolation. The segmentation principles are to guarantee 
the accuracy of the model topology and to ensure the tetrahedral attribute is single after boundary segmentation.

The segmentation method includes three steps: edge interpolation, face segmentation and tetrahedron segmentation.

(1)Edge interpolation

We use $T\left(d_{1} d_{2} d_{3} d_{4}\right)$ to denote the tetrahedron, use $d_{1}\left(x_{1}, y_{1}, z_{1}\right) 、 d_{2}\left(x_{2}, y_{2}, z_{2}\right)$ to denote two vertices of the tetrahedron and use $d_{12}\left(x_{12}, y_{12}, z_{12}\right)$ to denote the segmentation point. Assuming that the attributes of these two vertices $\left(d_{1}\left(x_{1}, y_{1}, z_{1}\right) 、 d_{2}\left(x_{2}, y_{2}, z_{2}\right)\right)$ are different, the segmentation point $\left(d_{12}\left(x_{12}, y_{12}, z_{12}\right)\right)$ is obtained by interpolation according to the gray values of the two points, the interpolation formula is shown in formula (1).

$$
f\left(d_{12}\right)=f\left(d_{1}\right)+\frac{f\left(d_{1}\right)-f\left(d_{2}\right)}{\left|d_{1}-d_{2}\right|}\left|d_{12}-d_{1}\right|
$$

We insert face segmentation point according to the edge segmentation point. There are two conditions, as shown in Figure 5: the colorful dots represent different organization attributes and the black dots represent the boundary segmentation points. In this paper, we select the midpoint of the triangle side to draw the Figure5.

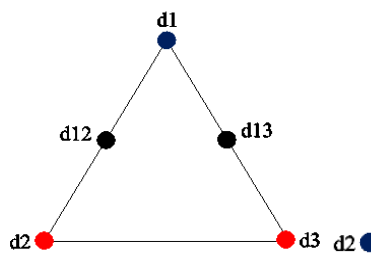

(a)

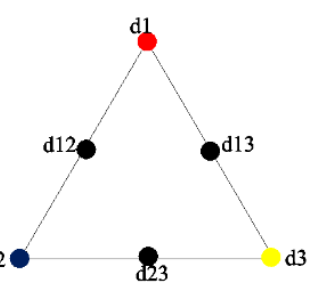

(b)

Figure 5. Segmentation Point of a Triangle

(2) Face segmentation

There are two possible ways to divide the triangular face into a single attribute triangular face after the segmentation points are inserted, as is shown in Figure 6.

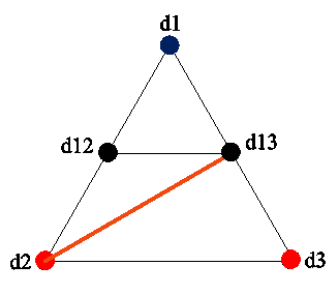

(a)

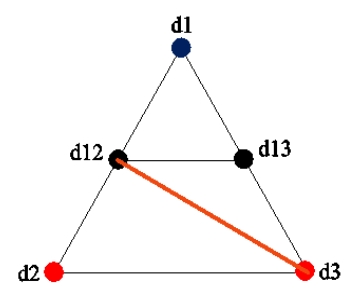

(b)

Figure 6. Two Segmentation Methods of a Triangle

The model topology will be wrong if we select a random segmentation method. In order to ensure the accuracy of model topology after segmentation, aiming at the triangular face shown in Figure 5 (a), we use the minimal number algorithm to select the vertex with the minimal number of connection points as a starting point and connect it to its opposite interpolation point.

The interpolation point of triangular face (Figure $5(\mathrm{~b})$ ) is shown in Figure 7. With this segmentation method, the attribute of the triangle face (denoted by $F\left(d_{12}, d_{13}, d_{23}\right)$ ) cannot be determined. For this, we insert an interpolation point (denoted by $d_{123}$ ) as a face segmentation point. In order to simplify the calculation, we denote point $d_{123}$ as the center 
of gravity of the face $F\left(d_{12}, d_{13}, d_{23}\right)$. And then, according to the segmentation method shown in Figure 7 (b) to guarantee the kind of the tetrahedron attribute is single after segmentation.

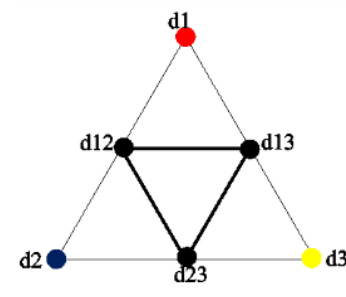

(a)

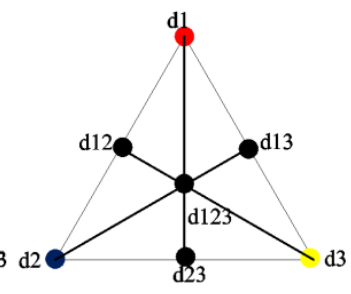

(b)

Figure 7. Two Segmentation Methods of a Triangle

Tetrahedron segmentation point insertion: this method is similar to the face segmentation that inserts tetrahedron segmentation points when the four vertices of the tetrahedron are different.

(3)Tetrahedron Division

According to segmentation points and the connection mode of our previous paper, the four types of tetrahedron are segmented successfully and the tetrahedron segmentation method is shown in Figure 8. The segmented tetrahedrons in Figure 8(a, b, c, d) are corresponding with the four types of tetrahedron which need to be segmented (Figure 4 (f, $\mathrm{g}, \mathrm{h}, \mathrm{j})$ ).

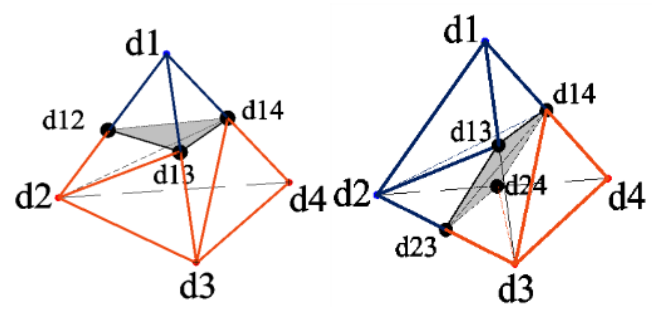

(a)

(b)

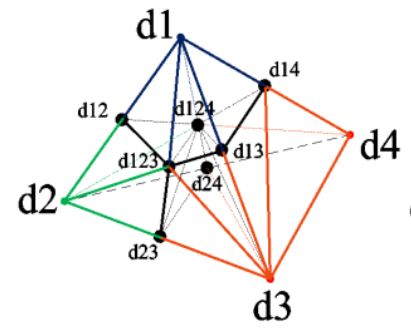

(c)

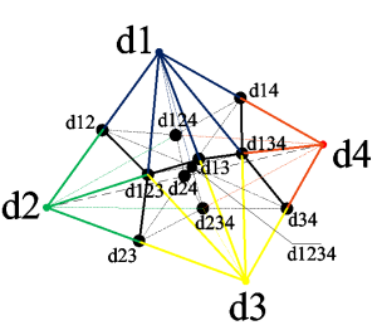

(d)

Figure 8. Segmentation Methods of a Tetrahedron

The colorful dots represent the vertices with one kind of attribute and the black dots represent the segmentation points, as are shown in Figure 8. After the tetrahedron segmentation, the attributes of tetrahedrons which are segmented are determined by using the tetrahedron attribute determination method in our previous paper. After all the attributes of tetrahedrons are determined, we obtain the core multi-organization model which has single attribute. 


\subsection{Model Information Visualization}

In this paper, we establish the view class in the MFC framework and encapsulate the OpenGL function in the View class to display the digital model.

Each element in the model is composed of point, line, triangular face and circumsphere. We use different colors to display the model elements according to the characteristics of multi-organization model. Therefore, we add new parameters which represent organization attribute into the relevant drawing functions of OpenGL to distinguish different multi-organization models by displaying different colors.

The model visualization can be realized by displaying each element after reading the data structure of the model successively.

\subsection{Model Visualization Interactive Operation}

Because of the complex structure of core space that contains a lot of little details, the model structure can't be fully observed by simple methods of model display. In this software, the users can observe model comprehensively by some interactive operations such as rotation, translation and scaling.

There are two methods to realize the model transformation in OpenGL: model transformation method and viewpoint transformation method. The former method has a fixed viewpoint and the geometric model can be multiplied by a transformation matrix by using this method; the latter method has a fixed model and the view point can be operated to display the model. In this paper, the viewpoint transformation method is used to realize the interactive operation of the three-dimensional model.

In order to observe the internal structure of core model more conveniently, we proposed a cutting display method to show the model. The method is as follows:

(1) According to the coordinates of model vertices, the bounding box of the model is calculated;

(2) Extracting the maximum value (which is denoted by $Z_{\max }$ ) and minimum value (which is denoted by $Z_{\text {min }}$ ) of the $\mathrm{Z}$ coordinates of the bounding box;

(3) The percentage value (which is denoted by $w$ ) defined by control slider;

(4) Defining the height of the cutting plane (which is denoted by $H_{c u t}$ ) in the direction of the $\mathrm{Z}$ axis, and the calculation formula is shown in formula (2):

$$
H_{\text {cut }}=Z_{\min }+\frac{\left(Z_{\max }-Z_{\min }\right) \times w}{100}
$$

(5) Judging whether the $\mathrm{Z}$ value (which is denoted by $\mathrm{Z}$ ) of the vertex coordinates of the tetrahedron element is less than $H_{c u t}$ or not. If $\mathrm{Z}<H_{c u t}$, then display model; if $\mathrm{Z}>H_{c u t}$, then do not display model.

\section{Modeling Examples of Multi-Organization Core}

The multi-organization model of a volcanic rock sample has been successfully reconstructed by using the multi-organization core modeling software.

The CT images of the volcanic rock (shown in Figure 9) are obtained by Sanying Precision Instruments Ltd. 


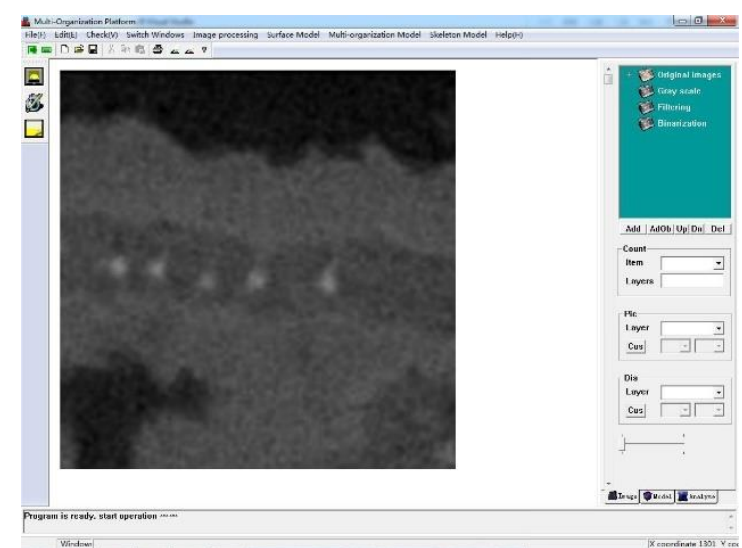

\section{Figure 9. CT Image of Volcanic Rock in a Certain Area}

In this paper, in order to simplify the process, we select a part of the CT images to reconstruct the multi-organization core model. As is shown in Figure10, Figure10 (a) shows the original core CT images; Figure10 (b) shows the images after noise reduction; Figure11 (c) shows the segmentation images after multi-organization division by using watershed algorithm; Figure10 (d) shows the acquisition voxels of multi-organization core.

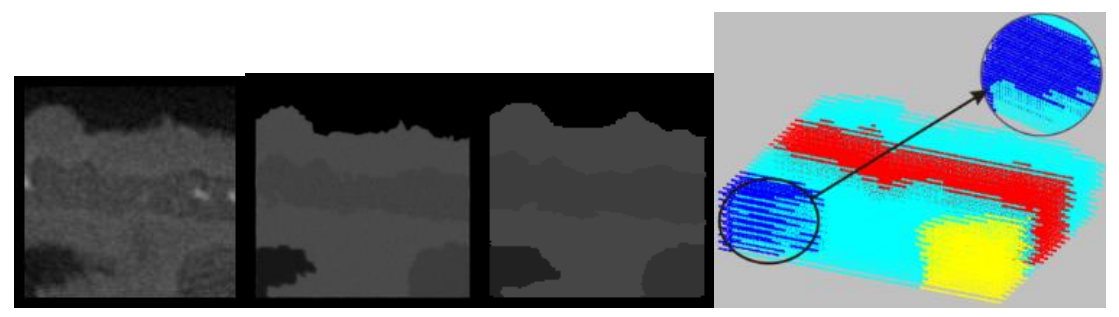

(a)

(b)

(c)

(d)

Figure 10. Acquisition of Multi Organization Data

The surface model which is established by MC algorithm is shown in Figure11.

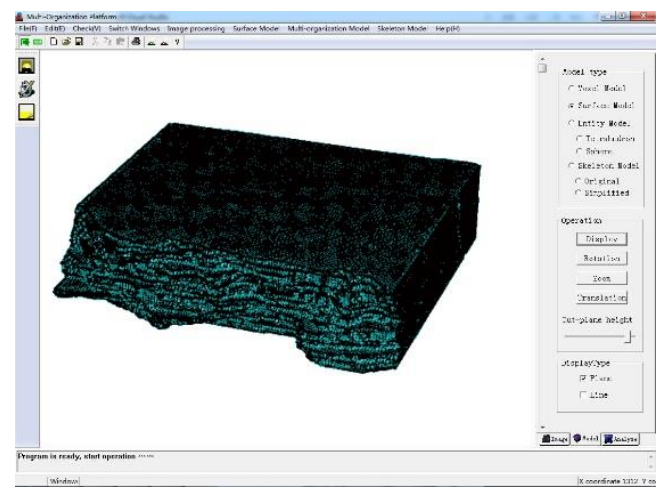

Figure 11. Surface model of Core

The tetrahedral core model which is constructed by constrained Delaunay triangulation algorithm is shown in Figure12. 


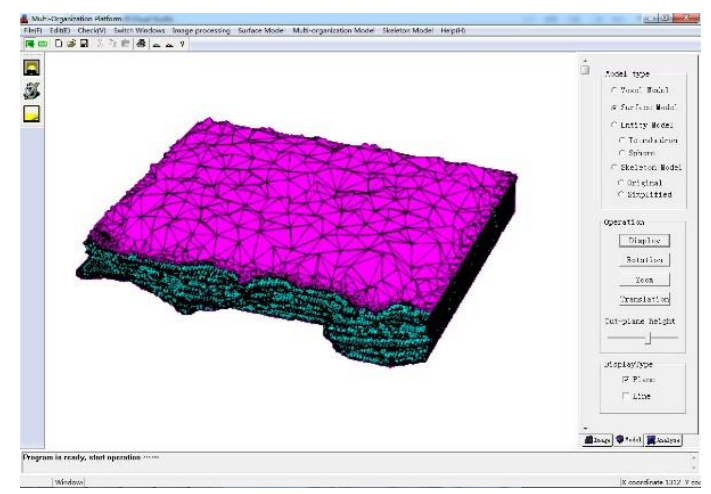

Figure 12. Tetrahedral Model of Core

After the tetrahedral core model constructed, we label the voxels to interpolation and the interpolation result is shown in Figure 13: the colorful parts of the Figure are a tetrahedral element with a single attribute and the white part is multi-organization boundary which should be divided into single attribute organizations.

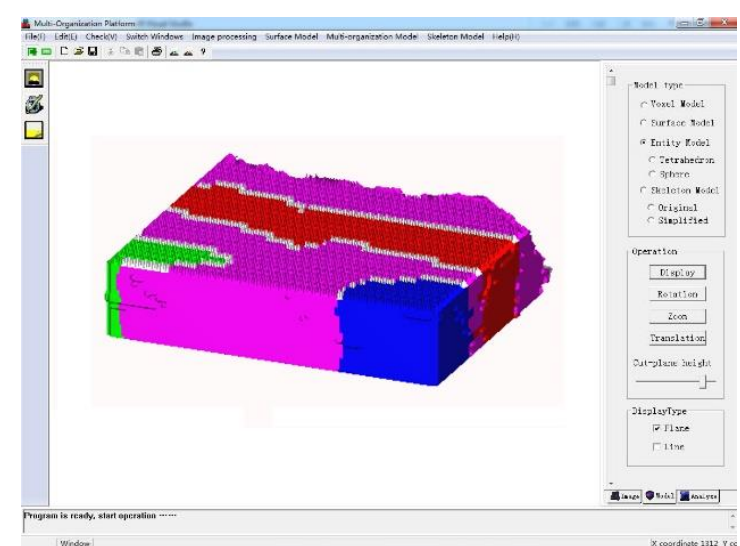

\section{Figure 13. Tetrahedron with Different Property}

Finally, the multi-organization boundary segmentation is realized by using the method which is proposed in the previous paper and the result is shown in Figure 14.

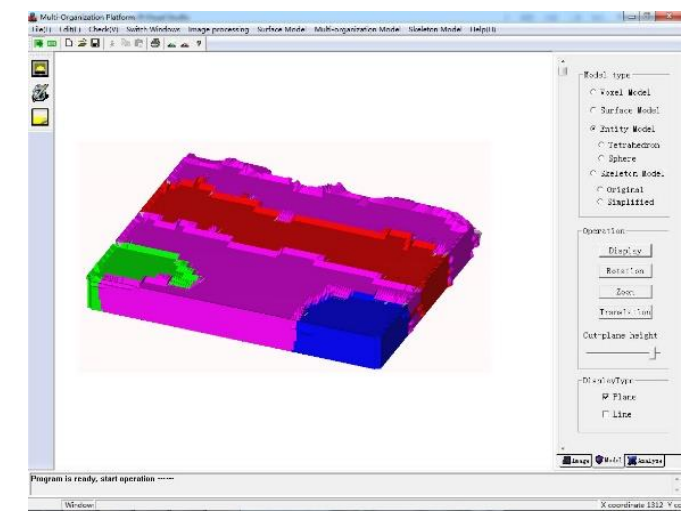

Figure 14. Segmentation Results

As shown in Figure 15, there are single organizations extracted from the multiorganization core model which can be analyzed separately. 


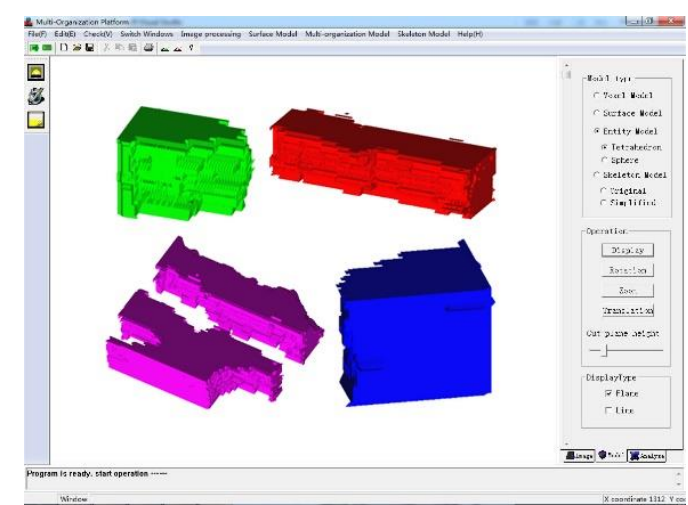

Figure 15. Result of Tetrahedron Dividing

\section{Conclusion}

In this paper, we present a voxels interpolation modeling method for multiorganization core that can reconstruct multi-organization core model conveniently and efficiently. On the basis of the method, we develop a multi-organization core modeling software based on C++ language and OpenGL display technology thatcan realize model visualization and interactive operation.

Our research is based on the medical multi-organization modeling method, aiming at the complex structures of the core, we develop and optimize this method to extract the single component of core which provides data support for the subsequent numerical analysis and simulation experiments.

\section{References}

[1] $\mathrm{Hu}$ Ping and LouXinyuan, "The development and application of multi organization grid modeling technology for medical images", China Science and technology, no. 11, (2009), pp. 134-134.

[2] L. Carl,J. Anand, D. Ivo, V. Lumitit, T, Arthur andV. H. John., "The generation of tetrahedral mesh models for neuroanatomical MRI", Neuroimage, vol.55, no.1, (2010), pp. 153-64.

[3] Yang Xiaosong, Shenhao, TangZesheng, "Segmented volume based tetrahedralization algorithm", Journal of Image and Graphics, vol.7, no.9, (2002), pp. 865-870.

[4] Feng Zhou, LiuRuilin, Ying Hailing,He Feng, Xiao Honglin, "Comparison study on the results of the core CT scan image segmentation and calculation of fracture porosity and logging data processing", Journal of Oil and Gas Technology, vol.33, no.4,(2011), pp. 100-104.

[5] Zhao Xiucai. "A dissertation submitted for the degree of doctor of philosophy", Shandong: China University of Petroleum (East China), (2009) (in Chinese).

[6] LiFangfang, YangShenglai, GaoWanglai, Ge Linwen, Chen Hao, Zhang Xing, "Research and application of large size quartz sand epoxy resin cement artificial core", Science Technology and Engineering, vol.13, no.3, (2013), pp. 685-689.

[7] Yang Wei, Zhu Min, LiMingzhao, Gao Hongbo,"Advanced merge based watershed image segmentation", Journal of Sichuan University(Natural Science Edition), vol.50, no.5, (2013), pp. 967972.

[8] WuHaibo, "Image segmentation algorithm based on watershed and level set method", Hunan: Central South University, (2009)(in Chinese).

[9] Lorensen, By W, and H. Cline, "Marching cubes: a high resolution 3d surface reconstruction algorithm", Computer Graphics, (2010).

[10] Z. Zhengyou, "Iterative point matching for registration of free-form curves and surfaces", International Journal of Computer Vision, vol.13, no.2, (1994), pp. 119-152.

[11] LI Wei, Han Lihua, Chen Jiaxin, JiZhihang, "A three dimensional reconstruction algorithm of fault medical image with auxiliary contour", Journal of Computer Applications, vol.28, no.9, (2008), pp. 2278-2280.

[12] Wang Lujun, "Research on three dimensional reconstruction of medical image", Chengdu: University of Electronic Science and Technology of China, (2004) (in Chinese).

[13] Yang Chenying, ChenWenliang. "Algorithm for 3D constrained Delaunay tetrahedral mesh division", Manufacture Information Engineering of China, vol.38, no.4, (2009), pp. 49-50. 
[14] HU Jianwei, FangLincong, LiuLigang, Wang Guozhao,"Mesh segmentation based on 3D delaunay triangulation”, Journal of Computer-Aided Design \& Computer Graphics, vol.21, no.12, (2009), pp. 1729-1732.

[15] Shang You, Editor, “OpenGL graphics programming guide”, China Water Conservancy and Hydropower Press, China, (2001).

[16] Sun Baoguang, "Study on visual simulation network software system of rod pumping", Shandong: China University of Petroleum (East China), (2014) (in Chinese).

\section{Authors}

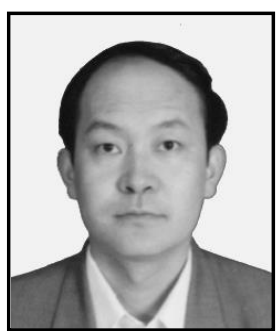

Niu Wenjie, received the Ph.D. degree in 2002 from Beihang University. He is a professor at China University of Petroleum (East China). His research interests include Visualization, computer graphics, oil drilling equipment design, etc. 
International Journal of Multimedia and Ubiquitous Engineering Vol.12, No.4 (2017) 